\title{
SO(2)-Networks as Neural Oscillators
}

\author{
Frank Pasemann, Manfred Hild, Keyan Zahedi \\ Fraunhofer Institute for Autonomous Intelligent Systems (AiS) \\ Schloss Birlinghoven, D-53754 Sankt Augustin, Germany \\ frank.pasemann, manfred.hild, keyan.zahedi@ais.fraunhofer.de
}

\begin{abstract}
Using discrete-time dynamics of a two neuron network with recurrent connectivity it is shown that for specific parameter configurations the output signals of neurons can be of almost sinusoidal shape. These networks live near the Sacker-Neimark bifurcation set, and are termed SO(2)-networks, because their weight matrices correspond to rotations in the plane. The discretized sinus-shaped waveform is due to the existence of quasi-periodic attractors. It is shown that the frequency of the oscillators can be controlled by only one parameter. Signals from the neurons have a phase shift of $\pi / 2$ and may be useful for various kinds of applications; for instance controlling the gait of legged robots.
\end{abstract}

\section{Introduction}

Aspects of discrete-time dynamics of two neuron networks with recurrent connectivity have been studied for a long time, e. g. [5], [8], [1], [2], [6]. This is because they are the simplest neural networks having non-trivial dynamical properties: for certain parameter domains one finds not only stationary attractors but oscillations of various periodicity, quasi-periodic and chaotic dynamics. There are various hysteresis phenomena observable, and different bifurcation scenarios involved. Most of these complex dynamical properties can be observed for recurrently coupled inhibitory and excitatory neurons with appropriate selfconnections [6].

Biologically this setup was motivated, among others, by the Wilson-Cowan model of neural populations [9]. Therefore it is assumed that the analysis of dynamical properties of 2-neuron networks can help to understand and explain phenomena observed in artificial recurrent networks in general, and possibly also those observed in biological neural systems. Furthermore, if one is interested more in the qualitative dynamical aspects of recurrent neural networks, than it is appropriate to study discrete-time dynamics, because it reflects all of those properties, which can be observed also for some (in general higher-dimensional) continuous-time dynamical systems (probably with time-delays).

The parameterization of these systems (synaptic weights, "slow" external inputs) allows to study the appearance and destruction of attractors, the delicate balance of stability and instability, features which seem to be crucial for many of the adaptive and higher-information processing capabilities of biological 
systems. A better understanding of these principles, eventually will allow alternative design methods, for instance robust neural controllers [3], in comparison to standard (and often not very efficient) learning algorithms.

Oscillatory dynamics in biological and artificial systems is of general interest, e.g. associated with various kinds of central pattern generators. But often smooth oscillations are desired, for instance when driving the legs of walking machines. For discrete-time dynamical systems, the sinusoidal shape of neural output signals is in general associated with appropriate quasi-periodic attractors. For 2-dimensional systems quasi-periodic orbits are dense on attractors which are compact 1-dimensional manifolds homeomorphic to a circle. They do appear after the system has passed a so-called Neimark-Sacker bifurcation [7]. For this reason, networks "living" near the Neimark-Sacker bifurcation set have been studied e.g. in [2], showing that the frequency of such systems can be controlled by external inputs. Here we choose a special type of 2-neuron network with this property, the weight matrix $w$ of which corresponds to a rotation in the plane; i.e. the matrix $w$ is an element in the special orthogonal group $\mathrm{SO}(2)$.

If an attractor of a 2-dimensional system is a perfect circle (e.g. harmonic oscillator), the resulting motion is called harmonic; the neural output then will correspond to a perfect sine wave. To describe the deviation from this perfect waveform we introduce a special measure of harmonicity for $\mathrm{SO}(2)$-networks, and study frequency and harmonicity of the oscillations in dependence of the rotation angle and a scaling factor.

\section{Two neuron networks}

In the following the discrete-time dynamics of two neuron networks with standard additive neurons is discussed. In general it is given by a 6 -parameter family of maps $f_{\rho}: \mathbf{R}^{2} \rightarrow \mathbf{R}^{2}, \rho=\left(\theta_{1}, w_{12}, w_{11}, \theta_{2}, w_{21}, w_{22}\right) \in \mathbf{R}^{6}$, where $\theta_{i}$ denotes the bias term of neuron $i$, and $w_{i j}$ the synaptic weight from neuron $j$ to neuron $i$. The output of a neuron is in general given by a sigmoidal transfer function $\sigma$, which here is chosen to be the hyperbolic tangent $\sigma=\tanh$. The presented results will also hold for other types of sigmoids. In fact, choosing the standard sigmoid $\sigma(x)=\left(1+e^{-x}\right)^{-1}$ will give networks with topologically equivalent dynamics. This is due to the relation $\tanh (x)=2 \cdot \sigma(2 x)-1$ and to a transformation of the corresponding parameters $\theta_{i}$ and $w_{i j}$, as was shown in [2], [6].

Furthermore, for convenience we will set $\theta_{1}=\theta_{2}=0$ in the following. The resulting two neuron dynamics is then given by the equations

$$
\begin{aligned}
& a_{1}(t+1):=w_{11} \sigma\left(a_{1}(t)\right)+w_{12} \sigma\left(a_{2}(t)\right), \\
& a_{2}(t+1):=w_{21} \sigma\left(a_{1}(t)\right)+w_{22} \sigma\left(a_{2}(t)\right) .
\end{aligned}
$$

where $a_{i}$ denotes the activity of neuron $i$.

Following the standard procedure, first the stability properties of fixed points

$$
a_{i}^{*}=\sum_{j=1}^{2} w_{i j} \sigma\left(a_{j}^{*}\right), \quad i=1,2 .
$$


have to be analyzed. Recall that the origin $a^{*}=(0,0)$ is always a fixed point of the dynamics (1). A fixed point $a^{*}$ is asymptotically stable if the eigenvalues of the Jacobian $D f_{\rho}\left(a^{*}\right)$ of the dynamics $f_{\rho}$ at $a^{*}$ all have modulus less than one. The Jacobian $D f_{\rho}\left(a^{*}\right)$ of the dynamics (1) is given by

$$
D f_{\rho}\left(a^{*}\right)=\left(\begin{array}{ll}
w_{11} \sigma^{\prime}\left(a_{1}^{*}\right) & w_{12} \sigma^{\prime}\left(a_{2}^{*}\right) \\
w_{21} \sigma^{\prime}\left(a_{1}^{*}\right) & w_{22} \sigma^{\prime}\left(a_{2}^{*}\right)
\end{array}\right) .
$$

Stability criteria for stationary states $a^{*}$ can be efficiently discussed in terms

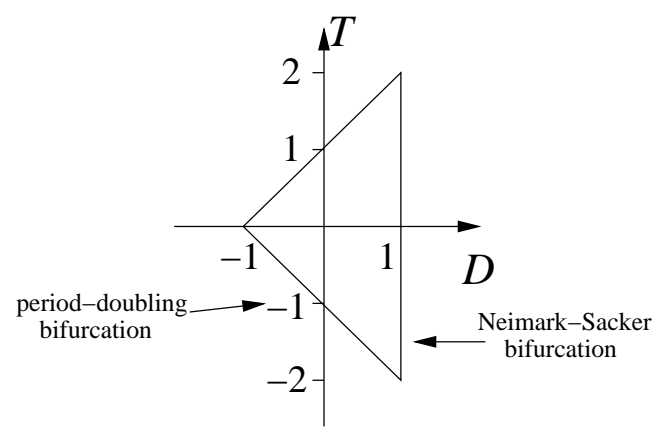

Fig. 1. The stability domain for a fixed point in terms of trace $\mathcal{T}$ and determinant $\mathcal{D}$ of the Jacobian $D f_{\rho}$.

of the trace $\mathcal{T}$ and the determinant $\mathcal{D}$ of $D f_{\rho}\left(a^{*}\right)$, which are here given by

$$
\mathcal{T}=w_{11} \sigma^{\prime}\left(a_{1}^{*}\right)+w_{22} \sigma^{\prime}\left(a_{2}^{*}\right), \quad \mathcal{D}=\left(w_{11} w_{22}-w_{12} w_{21}\right) \sigma^{\prime}\left(a_{1}^{*}\right) \sigma^{\prime}\left(a_{2}^{*}\right) .
$$

The eigenvalues of $D f_{\rho}\left(a^{*}\right)$ are then determined to be

$$
\lambda_{1,2}=\frac{1}{2}\left(\mathcal{T} \pm \sqrt{\mathcal{T}^{2}-4 \mathcal{D}}\right)
$$

The domain of stability for a fixed point $a^{*}$ in the $(\mathcal{T}, \mathcal{D})$-plane is given by a triangle bounded by the three straight lines $\mathcal{T}-\mathcal{D}=1, \mathcal{T}+\mathcal{D}=-1$, and $\mathcal{D}=1$ [7]. For instance, along the line $T+D=-1$ there will be a period-doubling bifurcation from a fixed point attractor to a period-2 attractor; along the line $\mathcal{D}=1,|\mathcal{T}|<2$ there will be Neimark-Sacker bifurcations from a fixed point attractor to a highly periodic or quasi-periodic attractor [7]. Of course, this last type of bifurcation is interesting because of the different types of oscillations one has to expect for configurations where the determinant $\mathcal{D}$ at a fixed point $a^{*}$ is larger than one.

Choosing as transfer functions tanh and setting the bias terms $\theta_{i}=0, i=$ 1,2 , will result in the fact that the origin $a^{*}=(0,0)$ is always a fixed point for

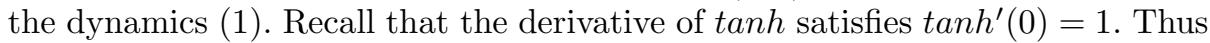
the Jacobian $D f_{\rho}(0)(3)$ is identical with the weight matrix $w=\left(w_{i j}\right)$ of the network. 


\section{$3 \quad \mathrm{SO}(2)$-networks}

Having identified the Jacobian $D f_{\rho}(0)$ at the origin with the weight matrix $w$ of the network, it is now easy to construct networks which correspond to configurations guaranteeing that the origin as a fixed point attractor undergoes a Neimark-Sacker bifurcation. Such networks have a weight matrix $w$ satisfying $\operatorname{det} w=1$. A special type of matrices, satisfying this condition, are the elements of the special orthogonal group $\mathrm{SO}(2)$. They are associated with rotations in the plane and a standard representation of these elements is given in terms of $\sin (\varphi)$ and $\cos (\varphi)$ of the rotation angle $\varphi$. Thus, convenient weight matrices are of the form

$$
w=D f_{\varphi}(0)=\left(\begin{array}{ll}
w_{11} & w_{12} \\
w_{21} & w_{22}
\end{array}\right)=\left(\begin{array}{cc}
\cos (\varphi) & \sin (\varphi) \\
-\sin (\varphi) & \cos (\varphi)
\end{array}\right),
$$

and we now can consider the dynamics (1) as a one parameter family of maps with parameter $-\pi \leq \varphi \leq \pi$ :

$$
\begin{aligned}
& a_{1}(t+1):=\cos (\varphi) \tanh \left(a_{1}(t)\right)+\sin (\varphi) \tanh \left(a_{2}(t)\right), \\
& a_{2}(t+1):=-\sin (\varphi) \tanh \left(a_{1}(t)\right)+\cos (\varphi) \tanh \left(a_{2}(t)\right) .
\end{aligned}
$$

Networks with these weight matrices will always have the origin as a nonhyperbolic fixed point; i.e. the eigenvalues $\lambda_{1,2}$ of the Jacobian $D f_{\varphi}(0)$ satisfy $\left\|\lambda_{1,2}\right\|=1$; they are complex numbers $\lambda_{1,2}=\cos (\varphi) \pm i \sin (\varphi)$. Varying $\varphi$ from $-\pi$ to $\pi$ now will keep the determinant $\mathcal{D}=1$ and at the same time $\mathcal{T}$ will vary between -2 and 2 , so that one moves along the line $\mathcal{D}=1$ in $(\mathcal{T}, \mathcal{D})$-space (figure 1).

Because here one wants to obtain almost sinusoidal output signals from the network, quasi-periodic attractors are preferred. Therefore one has to go slightly beyond the line $\mathcal{D}=1$ crossing the Neimark-Sacker bifurcation set. To do this one may introduce a second parameter $\alpha>1$ to obtain $\mathcal{D}>1$ for the determinant of the Jacobian $D f_{\rho}(0)$, and the weight matrix of such a network is given by

$$
w=D f_{(\alpha, \varphi)}(0)=\left(\begin{array}{ll}
w_{11} & w_{12} \\
w_{21} & w_{22}
\end{array}\right)=\alpha \cdot\left(\begin{array}{cc}
\cos (\varphi) & \sin (\varphi) \\
-\sin (\varphi) & \cos (\varphi)
\end{array}\right),
$$

and the eigenvalues $\lambda_{1,2}$ of the Jacobian satisfy $\lambda_{1,2}=\alpha \cdot e^{ \pm i \varphi}$. For obvious reasons networks with a weight matrix of the type (7) will be called $S O(2)$ networks. The parameter $\alpha$ can also be understood as controlling the slope of the transfer function tanh; i.e. in equation 1 we may replace the hyperbolic tangent by the function $\tau_{\alpha}(x)=\tanh (\alpha \cdot x)$; i.e. $\tau_{\alpha}^{\prime}(x)=\alpha \tanh ^{\prime}(\alpha x)$.

Staying in the parameter domains beyond the Neimark-Sacker bifurcation set, the frequency of the oscillations will change with varying $\alpha$ and $\varphi$. As was discussed in [2], the overall width of possible frequency range increases with $\alpha$ and depends crucially on $\varphi$. With $\alpha=1.0+\epsilon$, and $\epsilon<<1$ amplitudes of oscillations will be small and the waveform almost sine-shaped. With growing $\epsilon$, i.e. growing amplitude the nonlinearities get more pronounced and waveforms get more and more distorted. This qualitative considerations are supported by the following numerical analysis. 


\section{Simulations}

To get a first impression about the systems dynamics we depict in figure 2 the $(\varphi, \alpha)$-parameter domains for higher periodic attractors, showing frequency locking domains around $\varphi=0.5 \pi, 1.0 \pi$ corresponding to strong resonances (gray areas) of order 4 and 2, respectively. Higher order resonances, for instance 8 at $\varphi=0.25 \pi, 0.75 \pi$, are observed for larger $\alpha$-values. Black domains indicate quasi-periodic attractors. Setting $\alpha=1.5$, this is for instance verified in figure 3 , where the largest Liapunov exponent $L 1$ is always zero outside the frequency locking domains. Also for $\alpha=1.5$, the $\varphi$-dependence of the frequency $\omega$ of oscillations, calculated by the number of zero passages, is depicted in figure 4 ; it is almost linear, with frequency locking around $0.5 \pi$ and $\pi$. Around $\varphi=0$ we have only decoupled or weakly coupled neurons with super-critical self-connections resulting in four possible fixed point attractors.

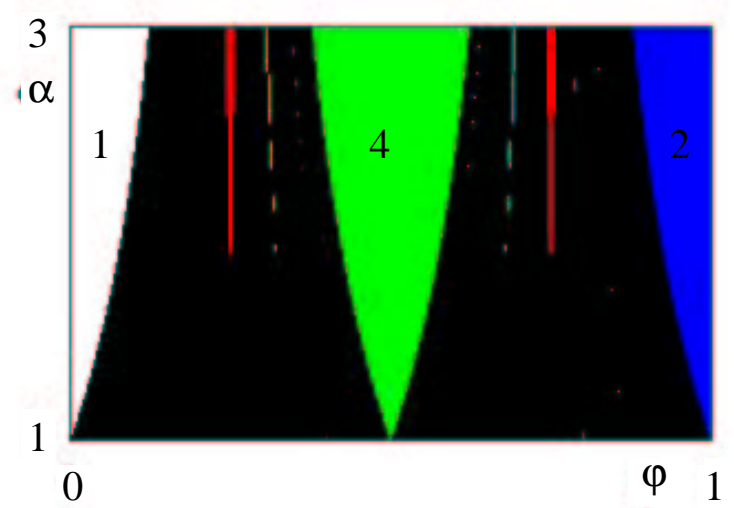

Fig. 2. $(\varphi, \alpha)$-parameter domains for quasi-periodic attractors (black), showing frequency locking domains around $\varphi=0.25 \pi, 0.5 \pi, 0.75 \pi$ and $\pi$.

That for small $\alpha$-values quasi-periodic attractors can be of almost circular shape is demonstrated in figure 5 for parameter values $\varphi=0.1 \pi$ and $\alpha=$ 1.05. Thus the dynamics corresponds to an almost harmonic motion, and output signals of the network have almost sinusoidal shapes as can be read from figure $5 \mathrm{~b}$.

With increasing amplitude of the oscillations, i.e. increasing $\alpha$, the wave shape will deviate from that of harmonic oscillation. To describe this the following measure of harmonicity $h$ for an attractor of the $\mathrm{SO}(2)$-systems is introduced:

$$
h:=\frac{\min (A)}{\max (A)}, \quad A=\left\{\|a(t)\| \mid t_{c} \leq t \leq t_{c}+N\right\},
$$

where $t_{c}$ denotes the number of convergent iterations, after which the dynamics is assumed to be near the attractor, and $\mathrm{N}$ is the number of counting iterations, 


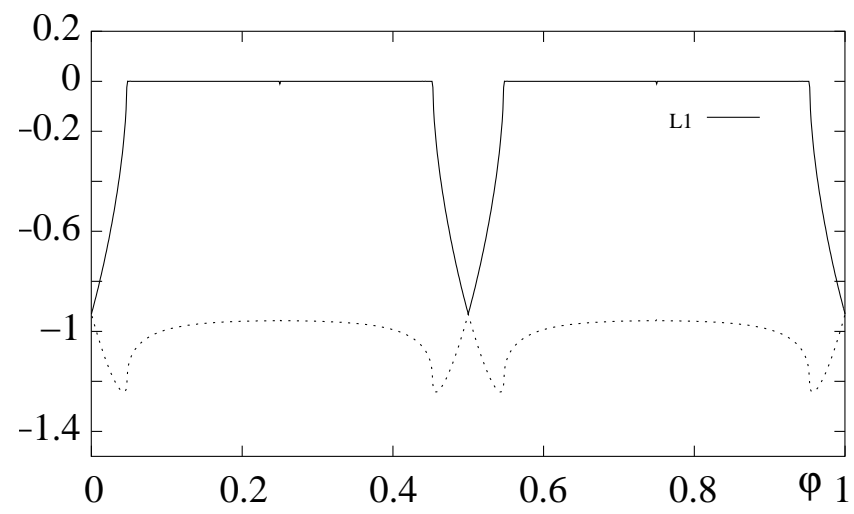

Fig. 3. Liapunov exponents for varying $\varphi$ and $\alpha=1.5$ fixed, showing that quasiperiodic attractors $(L 1=0)$ exist everywhere outside the frequency locking domains.

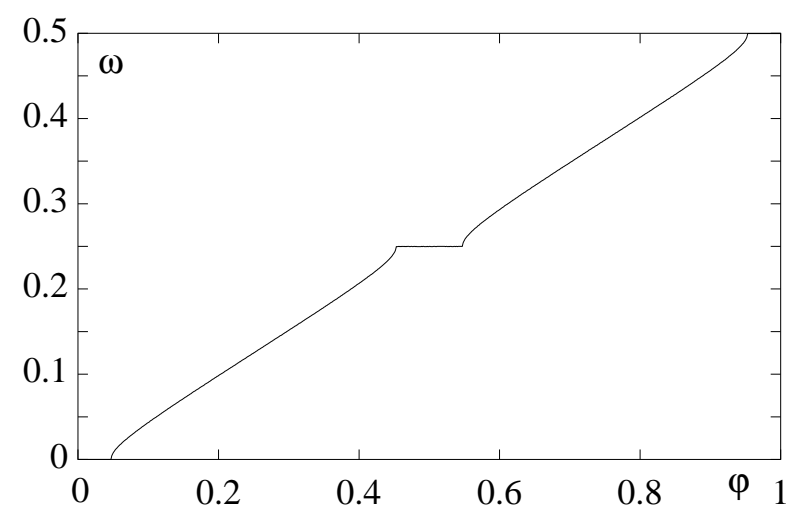

Fig. 4. Frequency $\omega$ depending on the rotation angle $0<\varphi<\pi$ for $\alpha=1.5$ fixed.

which should be large enough to represent a full period. Of course, $h=1$ describes a circle, and $h=1 / \sqrt{2}=0.707$ a perfect square. Fixing $\varphi$ one observes a strong dependence of the harmonicity $h$ of attractors on the parameter $\alpha$, as in figures 6 and 7; convergent and counting iterations were set to $t_{c}=N=5000$. For an angle $\varphi=0.1 \pi$ there is a smaller oscillatory $\alpha$-regime - as can be seen from figure 2 - over which harmonicity $h$ is decreasing with increasing $\alpha$. After it almost reaches the $h=0.707$, it suddenly jumps back to $h=1$ when the stable fixed point appears. At the same time the frequency is decreasing over this $\alpha$-interval (figure 6). For a larger angle $\varphi=0.3 \pi$ (figure 7) the oscillatory $\alpha$-regime is much larger, and harmonicity $h$ is decreasing in a highly nonlinear fashion with increasing $\alpha$. The frequency of oscillations stays almost constant over this $\alpha$-interval. Again, the decreasing harmonicity corresponds to attractors changing their shapes in output space from almost circular to almost quadratic. 

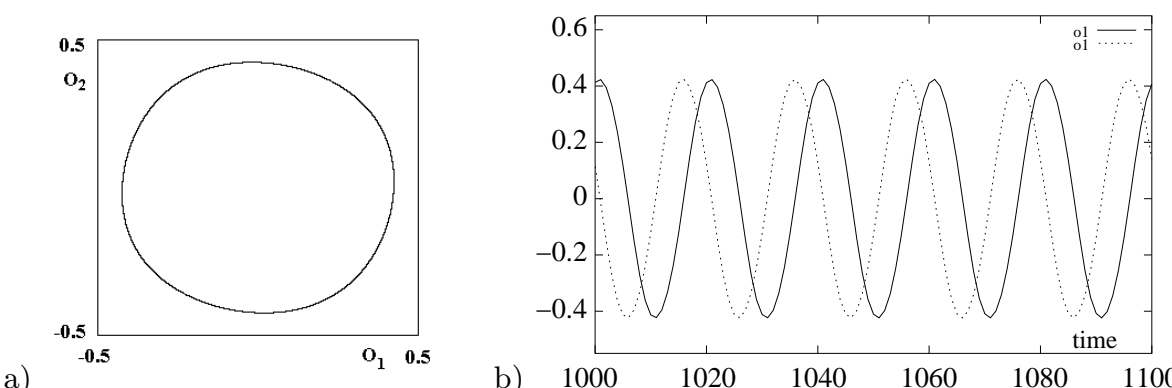

Fig. 5. a.) Attractor in $\left(o_{1}, o_{2}\right)$-space, and b) output signals of neurons 1 and 2 for $\alpha=1.05, \varphi=0.1 \pi$.

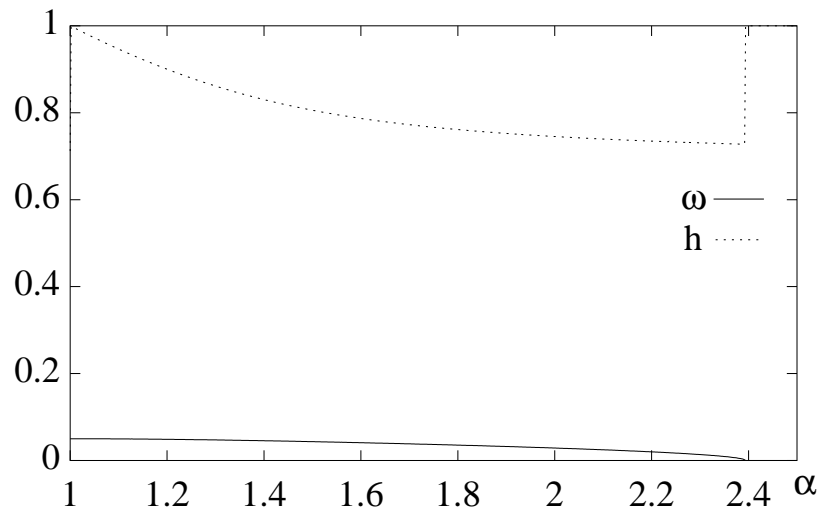

Fig. 6. Harmonicity $h$ of attractors in dependence of $\alpha$ with $\varphi=0.1 \pi$ fixed.

\section{Conclusions}

It was demonstrated that a specific class of 2-neuron networks, called $\mathrm{SO}(2)$ networks, can be used as frequency variable oscillators producing almost sinusoidal waveforms. The frequency can be controlled over a large domain mainly by the rotation angle $\varphi$ of of the $\mathrm{SO}(2)$ weight matrix, and the waveform, characterized by the harmonicity $h$ of the quasi-periodic attractor, can be adjusted mainly by the parameter $\alpha$ controlling the slope of the transfer function. Of course other variants of networks can be equally effective (see e.g. [2]), because parameters only have to guarantee that the determinant of the Jacobian is slightly larger than one. Therefore a convenient parameter may be also introduced as a modulation factor on one of the networks synaptic weights.

One possible application of these frequency variable oscillators is in the field of neural control for walking machines [4]. The described oscillators can control the type of walking and the walking speed of legged robots simply by using sensor inputs of the robot for weight or slope modulation. In addition, sensor inputs driving the neural system to and fro over the Neimark-Sacker bifurcation 


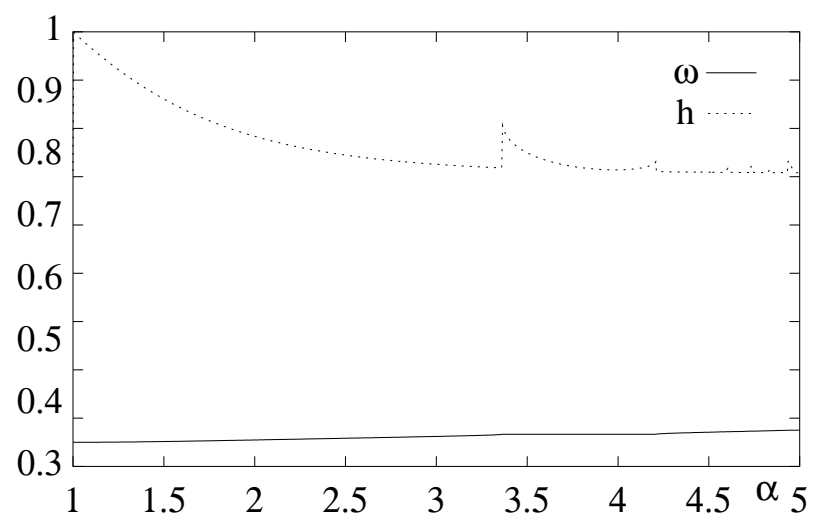

Fig. 7. Harmonicity $h$ of attractors in dependence of $\alpha$ with $\varphi=0.3 \pi$ fixed.

set will switch on and off the oscillations. Operating the neural oscillator near the bifurcation set, i.e. $\alpha=1+\epsilon, \epsilon<<1$, will cause long transients, and thus will result in a smooth type of switching. As an example, this will be demonstrated for a quadruped robot elsewhere.

\section{References}

1. Chapeau-Blondeau, F., and Chauvet G. (1992), Stable, oscillatory, and chaotic regimes in the dynamics of small neural networks with delay, Neural Networks, 5, $735-743$.

2. Haschke, R., Steil, J.J̃., and Ritter, H. (2001), Controlling oscillatory behaviour of a two neuron recurrent neural network using inputs, in: G. Dorffner, H. Bischof, K. Hornik (Eds.): Artificial Neural Networks - ICANN 2001, International Conference Vienna, Austria, August 21-25, 2001, Proceedings. LNCS 2130, Springer Verlag, Berlin, p. $1109 \mathrm{ff}$.

3. Hülse, M., and Pasemann, F. (2002) Dynamical neural Schmitt trigger for robot control, in: J.R. Dorronsoro (Ed.): Artificial Neural Networks - ICANN 2002, International Conference, Madrid, Spain, August 28-30, 2002. Proceedings. LNCS 2415, Springer Verlag, Berlin, pp. 783-788.

4. Kimura, H., Akiyama, S., and Sakurama, K. (1999), Realization of dynamic walking and running of the quadruped using neural oscillator, Autonomous Robots, $\mathbf{7}$, pp. $247-258$.

5. Marcus, C. M., and Westervelt R. M. (1989), Dynamics of iterated-map neural networks, Phys. Rev., A 40, 501-504.

6. Pasemann, F. (2002) Complex dynamics and the structure of small neural networks, Network: Computation in Neural Systems, 13, 195-216.

7. Thompson, J. M. T., and Stewart, H. B. (1986), Nonlinear Dynamics and Chaos, Chichester: John Wiley.

8. Wang, X. (1991) Period-doublings to chaos in a simple neural network: An analytic proof, Complex Systems, 5, 425-441.

9. Wilson, H. R., and Cowan,J. D. (1972), Excitatory and inhibitory interactions in localized populations of model neurons, Biophys. J., 12, 1-23. 